\title{
Retroperitoneal Bronchogenic Cyst Mimicking a Pancreatic Cystic Lesion with Extremely High Level of Intralesional Fluid CA-19.9 Antigen: Benign in Disguise
}

\author{
Jessé Clementino-Filho ${ }^{1} \cdot$ Rodrigo Cañada Trofo Surjan $^{2} \cdot$ Eloy Taglieri $^{1} \cdot$ José Celso Ardengh $^{1}$ (1)
}

Received: 8 April 2021 / Accepted: 25 September 2021 / Published online: 25 November 2021

(c) Association of Surgeons of India 2021

\begin{abstract}
Bronchogenic cysts are congenital benign tumors resulting from abnormal budding of the primitive foregut. Usually presented on the posterior mediastinum, its presence on the retroperitoneum is extremely rare. We present an asymptomatic lady patient with a retroperitoneal cystic lesion that was submitted to endoscopic ultrasound-guided biopsies and intracystic fluid aspiration with histology excluding malignance despite intracystic fluid biochemical analysis that disclosed extremely high carbohydrate antigen 19-9. Definite diagnosis of bronchogenic cyst was only possible after complete surgical resection of the lesion. Furthermore, we discuss the use of this antigen as a tumor marker in this situation and its relevance to the preoperative diagnosis of such lesions.
\end{abstract}

Keywords Bronchogenic cyst $\cdot$ Retroperitoneal neoplasms $\cdot$ CA-19-9 antigen $\cdot$ Endosonography

\section{Introduction}

First described by Meyer et al. in 1859 [1], bronchogenic cysts $(\mathrm{BC})$ are benign congenital malformations resulting from aberrant budding of the primitive foregut between the third and seventh gestational weeks. Most of BC originate in the posterior aspect of the superior mediastinum, being the most common mediastinal cysts, while up to $20 \%$ may occur within the lung parenchyma [2,3].

Retroperitoneal bronchogenic cysts are extremely rare and the first case report was published by Miller et al. in 1953 [1, 2]. Being asymptomatic lesions most of time, they are usually incidentally detected during routine image studies. Differential diagnosis with other retroperitoneal cystic tumors is challenging and usually reached after surgical resection and pathological examination of the surgical specimen $[1,2,4]$.

José Celso Ardengh

jcelso@uol.com.br

1 Gastrointestinal Endoscopy Service, Hospital Nove de Julho, Rua Peixoto Gomide, 645, Cerqueira César, São Paulo 01409-001, Brazil

2 Surgery Service, Hospital Nove de Julho, São Paulo, Brazil
We report a lady patient with asymptomatic retroperitoneal BC with highly elevated cystic fluid carbohydrate antigen 19-9 (CA-19-9) level that was submitted to surgical resection. We discuss the association of this lesion with cystic fluid and serum CA-19-9 elevation and its relevance as a tumor marker.

\section{Case Report}

A 66-year-old lady patient, previously asymptomatic and with medical history of systemic hypertension, diabetes mellitus, dyslipidemia and recently treated for COVID 19, was submitted to a contrast enhanced computed tomography of the abdomen due to abdominal pain after fall from standing. The image study disclosed an incidental $6.2 \times 3.0-\mathrm{cm}$ upper abdominal solid-cystic retroperitoneal lesion, with mild contrast enhancement and close to the esophagogastric transition, left diaphragmatic crus and left adrenal gland (Fig. 1). An angiotomography was performed and demonstrated the close relation of the tumor with the splenic artery $\left(<90^{\circ}\right)$ and the left gastric artery without clear tumor vascular involvement.

The patient was then submitted to a magnetic resonance image study that disclosed that the tumor had close anatomic relation with the esophagogastric transition, left adrenal 
Fig. 1 Computed tomography of the abdomen disclosing a $6.2 \times 3.0 \mathrm{~cm}$ solid-cystic retroperitoneal tumor, with mild contrast enhancement and intralesional calcification. a Close relation of the tumor (circled in red) with the left diaphragmatic crus; b Close relation of the tumor (circled in red) with the left adrenal gland
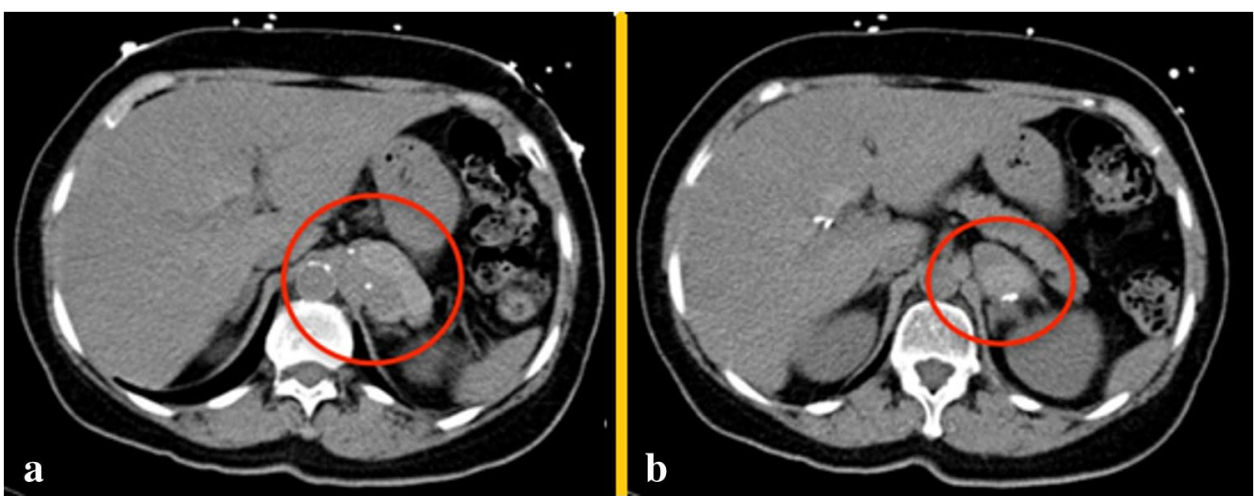

gland and the pancreatic body, without tumor involvement of these organs (Fig. 2).

At this time, serum tumor markers levels were CA-19-9: $16,4 \mathrm{U} / \mathrm{ml}$ (reference range: $<37 \mathrm{U} / \mathrm{ml}$ ), CEA: $<0.5 \mathrm{ng} / \mathrm{ml}$ (reference range for non-smoking adults: $<5 \mathrm{ng} / \mathrm{ml}$ ) and CA 125: $5.7 \mathrm{U} / \mathrm{ml}$ (reference rage: $<35 \mathrm{U} / \mathrm{ml}$ ).

An endoscopic sonography was performed and disclosed a 5-cm well-delimited solid-cystic lesion with intralesional calcifications in close relation to the splenic artery and the pancreatic body. The lesion was hypo-vascularized by sonographic Doppler evaluation and presented soft characteristics at qualitative elastography. Intracystic fluid aspiration and ultrasound guided biopsies with 20-gauge ProCore ${ }^{\circledR}$ EchoTip (Cook Medical, Limerick, Ireland) were performed (Fig. 3).

Biochemical analysis of the lesion fluid is demonstrated in Table 1 and showed extremely high levels of CA 19-9 and amylase on the fluid. Histologic examination of the biopsies presented macrophage aggregations without signs of malignancy being suggestive of a pancreatic pseudocyst.

Due to the uncertain diagnosis and the high level of intralesional tumor marker CA-19-9, surgical resection was proposed. The complete resection of the tumor with free surgical margins was performed. The lesion was dissected from the esophagogastric junction, left diaphragmatic crus and splenic artery. There were no adherences of the tumor with the left adrenal gland and pancreatic body (Fig. 4). Operative time was $4 \mathrm{~h}$ and estimated bleeding was $100 \mathrm{ml}$. Postoperative period was uneventful and the patient was discharged on the fourth postoperative day.

Histopathological surgical specimen examination showed islands of cartilage with calcification, smooth muscle tissue and cysts lined by pseudostratified columnar ciliated epithelium with sparse caliciform cells and serous-mucinous glands. This epithelium is similar to the epithelium coating the respiratory tree. Cystic walls contained macrophage aggregations. These findings permit the diagnosis of BC (Fig. 5). Immunohistochemistry test confirmed the bronchial origin of the epithelium, reinforcing the $\mathrm{BC}$ diagnosis (Table 2, Fig. 6).

\section{Discussion}

CA-19-9 is a carbohydrate antigen detected by the specific monoclonal antibody 116-NS-19-9, or Sialyl Lewis A IgG1, originally described in 1979 by the Polish-born virologist Hilary Koprowski while searching for an antigen unrelated
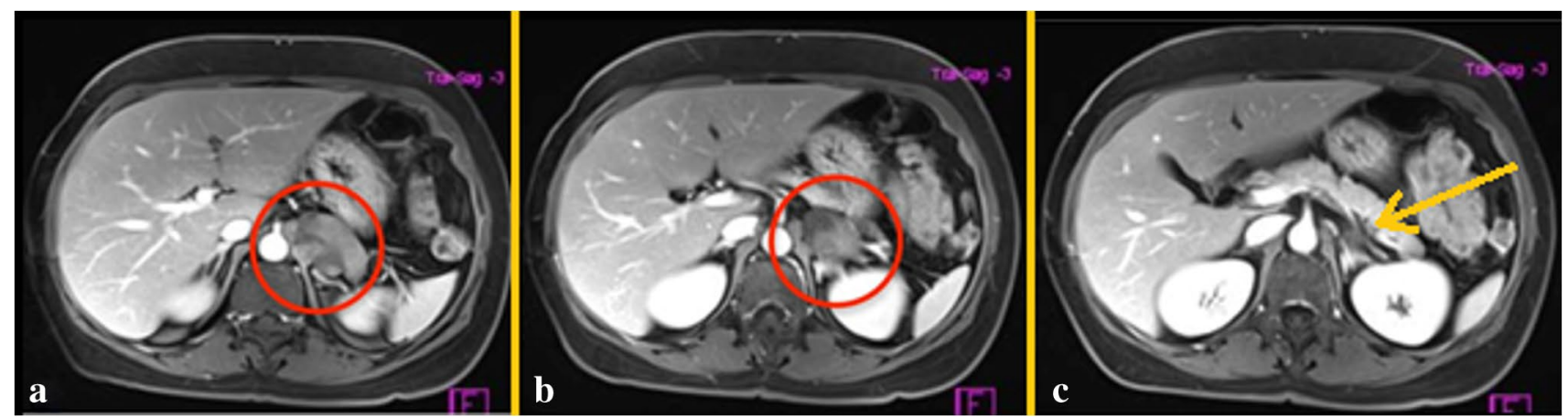

Fig. 2 Magnetic resonance of the abdomen disclosing heterogenous retroperitoneal lesion. a close anatomic relation of the lesion and the left diaphragmatic crus (circled in red); b left retroperitoneal tumor close to the left adrenal gland (circled in red); $\mathbf{c}$ tumor located posterior to the pancreatic body (yellow arrow) 
Fig. 3 Endoscopic sonography. a Well-delineated $5 \mathrm{~cm}$ solid-cystic lesion with intralesional calcifications with close relation with the splenic artery and pancreatic body; b hypo-vascularization of the lesion at doppler sonography; c soft characteristics of the tumor at qualitative elastography; $\mathbf{d}$ lesion ultrasound-guided biopsy of the

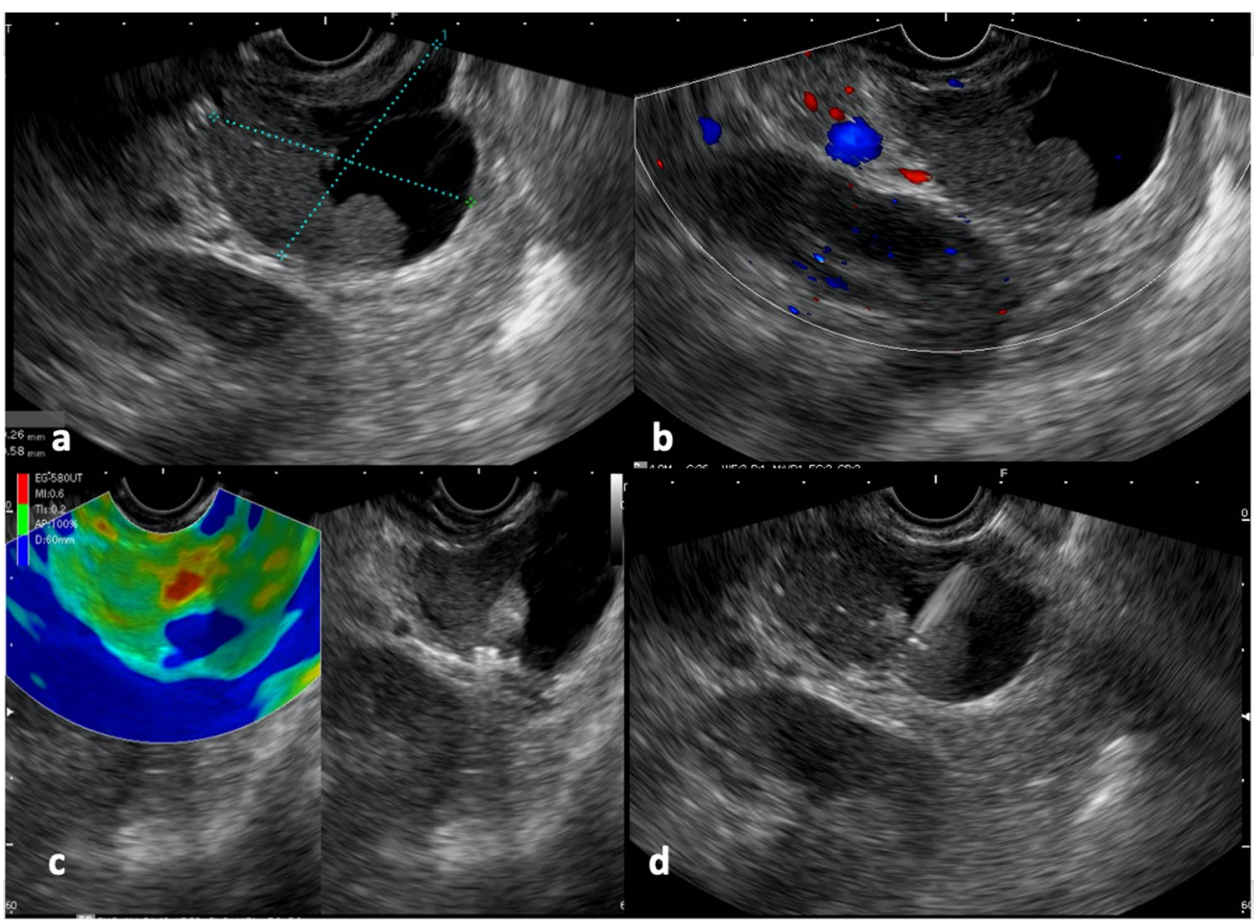

\begin{tabular}{ll}
\hline Amylase & $>50.000 \mathrm{U} / \mathrm{L}$ \\
\hline CEA & $56 \mathrm{ng} / \mathrm{mL}$ \\
CA-19-9 & $95.434,1 \mathrm{U} / \mathrm{mL}$ \\
Glucose & $1 \mathrm{mg} / \mathrm{dL}$ \\
\hline
\end{tabular}

Table 1 Biochemical analysis of the lesion fluid

to and more specific than carcinoembryonic antigen (CEA) in the serum of patients with colorectal cancer. The virologist had moved to the USA after World War II and was also responsible for the development of the first oral poliomyelitis vaccine $[5,6]$.

Commonly used as a tumor marker for biliopancreatic malignancies, CA-19-9 can also be elevated in the serum of patients with hepatocellular carcinoma, colorectal cancer, gastric cancer, gynecological malignancies, biliary obstruction, pancreatitis, diabetes mellitus, interstitial pulmonary disease, asbestosis, tuberculosis, rheumatologic diseases, excessive tea consumption and pulmonary neoplasia [7-10].

While often associated with several malignancies (specially biliopancreatic cancers), it is long known that CA-19-9 is commonly expressed in mucinous cells of bronchial glands and surface of the bronchiolar epithelium in different benign pulmonary diseases, resulting in serum levels of this antigen as high as in gastrointestinal malignancies [10].

Bronchogenic cysts are the most common congenital primary cysts of the mediastinum. Being derived from the ding of the tracheobronchial tree or lung bud between the 26th and 40th days of gestation. The result is a fluid-filled and blind-ended pouch [11]. Rarely, they migrate to atypical locations, including the below diaphragm, as a result of a pinching of the tracheobronchial tree during the fusion primitive foregut, it resulted from abnormal or late bud-

Fig. 4 Specimen. a macroscopic aspect of the lesion; $\mathbf{b}$ multilocular aspect of the lesion, cut in half

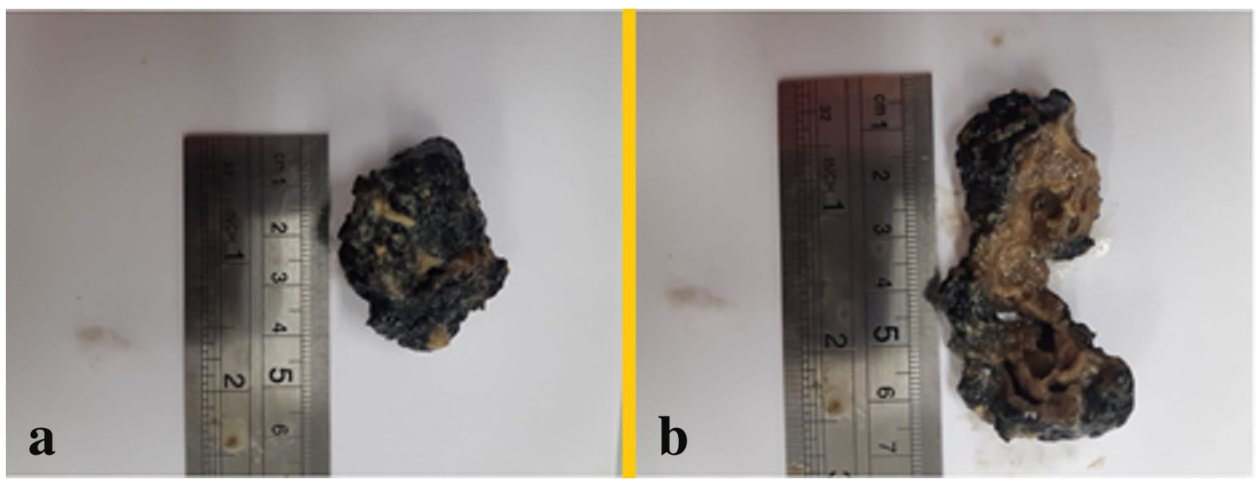



ings disclosing islands of cartilage with calcification, smooth muscle tissue and cysts lined by pseudostratified columnar ciliated epithelium with sparse caliciform cells and serousmucinous glands. a H\&E, $\mathrm{x} ; \mathbf{b}$ $H \& E$, x. (H\&E: hematoxylin and eosin stain)
Fig. 5 Histopathological find-

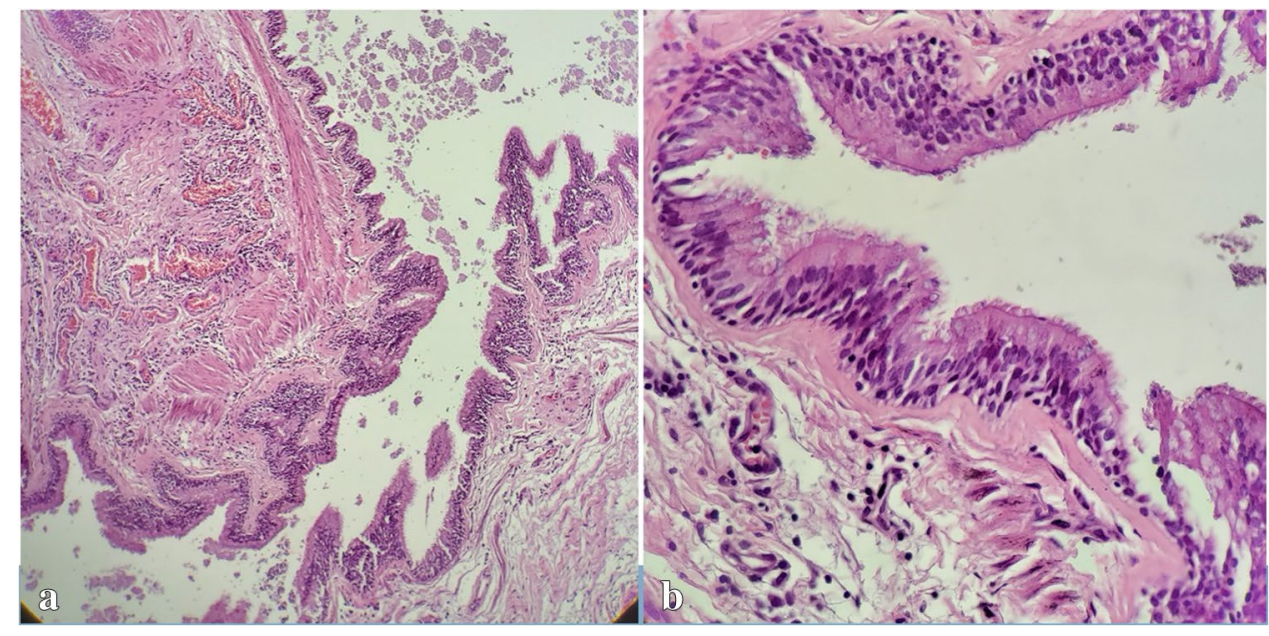

\begin{tabular}{ll}
\hline Antibodies & Staining \\
\hline CK7 & Positive \\
TTF1 & Positive \\
Napsyn & Positive \\
Amylase & Positive \\
Ki-67 & Positive; $1 \%$ \\
CK20 & Negative \\
CDX2 & Negative \\
MUC1 & Negative \\
MUC5AC & Negative \\
SATB2 & Negative \\
CA-19-9 & Negative \\
SALL4 & Negative \\
\hline
\end{tabular}

of the pleuroperitoneal membranes [12]. Retroperitoneal $\mathrm{BC}$ occur equally in men and woman, frequently on the upper left side of the retroperitoneum (82\% of cases) [13].

Although being benign lesions, surgical resection is usually performed, in order to reach definite diagnosis of the tumor and prevent complications. While preoperative diagnosis of $\mathrm{BC}$ represents a great challenge, the use of endoscopic ultrasonography has emerged as a useful tool [14]. In addition to providing anatomical information, it allows to perform endoscopic ultrasonography-guided biopsy of the lesion and cystic fluid aspiration for biochemical analysis.

In our reported case, the cystic fluid biochemical analysis demonstrated highly elevated CA-19-9 and amylase levels (Table 1). Therefore, to a lesion related to the pancreas with high biliopancreatic tumor marker content the first conduct thought was surgical resection for both treatment and definite diagnosis. Finally, pathology disclosed a benign retroperitoneal $\mathrm{BC}$, raising the question of the reasons why these lesions could content elevated fluid cyst CA-19-9 and amylase.
The first report regarding high intralesional fluid CA-19-9 on BC dates to 1989 by Okubo et al. [15]. In 1996, Ohkouchi et al. reported an interesting case of a patient with BC that presented with elevated serum CA-19-9 that fluctuated with cystic fluid volume [16]. Previous scientific experiments using hydrostatic pressure aiming to develop vaccines to different tumors by inducing in vitro tumor cells inactivation while maintaining cellular immunogenic features demonstrated that hydrostatic pressure results in release of cell-derived antigens that, ultimately, may enter the blood stream [17]. This may be the explanation for both the fluctuating serum levels of CA-19-9 of the patient reported by Ohkouchi et al. and the lack of CA-19-9 staining on immunohistochemical examination of the specimen of our patient despite the extremely elevated intralesional cystic fluid CA-19-9 content. The presence of immunoreactivity to amylase and its secretion in the bronchial ciliated epithelial cells and in serous cells of bronchial glands is also well known, which could explain the high intracystic levels and the positivity in immunohistochemistry to amylase in the case's lesion [18]. Therefore, despite the elevated levels of amylase and CA-19-9 on the cystic fluid obtained by endoscopic ultrasonography aspiration of the retroperitoneal solid-cystic lesion of our patient, these findings may indicate neither a malignant lesion nor any relation of the tumor with the pancreas.

\section{Conclusion}

Bronchogenic cysts are benign lesions that are difficult to diagnose without histological and immunohistochemical examination of the surgically resected specimen. The presence of this tumor on the retroperitoneum is extremely rare, and high suspicion is paramount to reach preoperative diagnosis. Although specially associated with biliopancreatic tumors, even extremely elevated cystic fluid and 


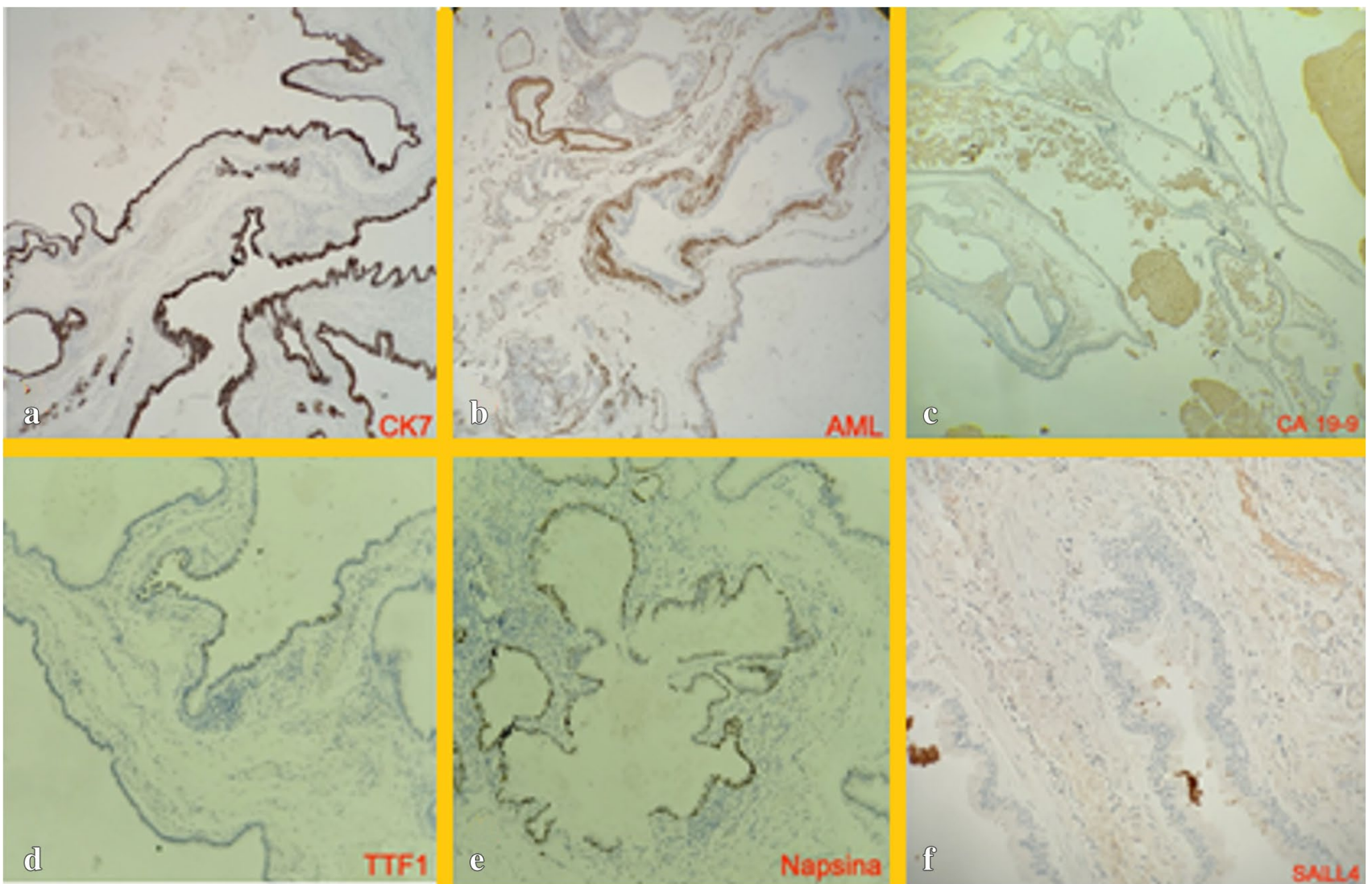

Fig. 6 Immunohistochemical tests. a CK7: positive; b amylase: positive; c CA-19-9: negative; d TTF1: positive; e napsin: positive; f SALL4: negative

serum CA-19-9 levels on patients with suspected bronchogenic cysts should not be used as a pathognomonic sign of malignancy.

\section{References}

1. Govaerts K, van Eyken P, Verswijvel G, van der Speeten K (2012) A bronchogenic cyst, presenting as a retroperitoneal cystic mass. Rare Tumors 4(1):37-44

2. Sarper A, Ayten A, Golbasi I, Demircan A, Isin E (2003) Bronchogenic Cyst. Tex Heart Inst J 30(2):105-108

3. Wang M, He X, Qiu X, Tian C, Li J, Lv M (2017) Retroperitoneal bronchogenic cyst resembling an adrenal tumor with high levels of serum carbohydrate antigen 19-9. Med (United States) 96(31):30-32

4. Runge T, Blank A, Schäfer SC, Candinas D, Gloor B, Angst E (2013) A retroperitoneal bronchogenic cyst mimicking a pancreatic or adrenal mass. Case Rep Gastroenterol 7(3):428-432

5. Koprowski H et al (1979) Colorectal carcinoma antigens detected by hybridoma antibodies. Somatic Cell Genet 5(6):957-971

6. Koprowski $\mathrm{H}$ et al (1981) Specific antigen in serum of patients with colon carcinoma. Science 212(4490):53-55

7. Ballehaninna UK et al (2011) Serum CA 19-9 as a biomarker for pancreatic cancer-a comprehensive review. Indian J Surg Oncol 2(2):88-100
8. Howaizi M et al (2003) A new cause for CA19.9 elevation: heavy tea consumption. Gut. 52(6):913-914

9. Sato Y, et al (2016) The prognostic value of serum CA 19-9 for patients with advanced lung adenocarcinoma. BMC Cancer 16: 890

10. Takayama S, Kataoka N, Usui Y, Inase N, Natori Y, Nakayama M, Miura H, Kimula Y (1990) CA 19-9 in patients with benign pulmonary diseases. Nihon Kyobu Shikkan Gakkai Zasshi 28(10):1326-1331

11. McAdams HP, Kirejczyk WM, Rosado-de-Christenson ML, Matsumoto S (2000) Bronchogenic cyst: imaging features with clinical and histopathologic correlation. Radiology 217(2):441-446. https://doi.org/10.1148/radiology.217.2.r00nv19441

12. Wen Y, Chen W, Chen J, He X (2020) Retroperitoneal bronchogenic cyst resembling an adrenal tumor: two case reports and literature review. J Int Med Res 48(5):0300060520925673

13. Chung JM, Jung MJ, Lee W et al (2009) Retroperitoneal bronchogenic cyst presenting as adrenal tumor in adult successfully treated with retroperitoneal laparoscopic surgery. Urology 73(442): e13-e15

14. Han C, Lin R, Jun Yu, Zhang Q, Zhang Y, Liu J, Ding Z, Hou X (2016) A case report of esophageal bronchogenic cyst and review of the literature with an emphasis on endoscopic ultrasonography appearance. Medicine (Baltimore) 95(11):e3111. https://doi.org/ 10.1097/MD.0000000000003111

15. Okubo A, Sone S, Ogushi F, Ogura T, Uyama T, Monden Y et al (1989) A case of bronchogenic cyst with high production of antigen CA 19-9. Cancer 63(10):1994-1997 
16. Ohkouchi M, Akizuki N, Jin Y, Ichioka M, Marumo F (1996) Bronchial cyst in a patient with a high serum CA19-9 concentration that fluctuated with the cyst fluid volume. Japanese J Thorac Dis 34(4):482-486

17. Weiss EM, Meister S, Janko C, Ebel N, Schlücker E, MeyerPittroff R, Fietkau R, Herrmann M, Gaipl US, Frey B (2010) High hydrostatic pressure treatment generates inactivated mammalian tumor cells with immunogeneic features. J Immunotoxicol 7(3):194-204. https://doi.org/10.3109/15476911003657414

18. Hayashi Y, Fukayama M, Koike M, Nakayama T (1986) Amylase in human lungs and the female genital tract Histochemical and immunohistochemical localization. Histochemistry 85(6):491496. https://doi.org/10.1007/BF00508431

Publisher's Note Springer Nature remains neutral with regard to jurisdictional claims in published maps and institutional affiliations. 